\title{
Protocol: Streamlined sub-protocols for floral-dip transformation and selection of transformants in Arabidopsis thaliana Amanda M Davis ${ }^{\dagger 1}$, Anthony Hall ${ }^{\dagger 2}$, Andrew J Millar ${ }^{3}$, Chiarina Darrah ${ }^{\dagger 1}$ and Seth J Davis*1
}

Address: ${ }^{1}$ Max Planck Institute, Cologne, Germany, ${ }^{2}$ University of Liverpool, Liverpool, UK and ${ }^{3}$ University of Edinburgh, Edinburgh, UK Email: Amanda M Davis - amdavis@mpiz-koeln.mpg.de; Anthony Hall - anthony.hall@liverpool.ac.uk; Andrew J Millar - andrew.millar@ed.ac.uk; Chiarina Darrah - Chiarina.Darrah@UGent.be; Seth J Davis* - davis@mpiz-koeln.mpg.de

* Corresponding author †Equal contributors

Published: 27 February 2009

Plant Methods 2009, 5:3 doi:10.1186/1746-48।I-5-3
Received: 9 December 2008

Accepted: 27 February 2009

This article is available from: http://www.plantmethods.com/content/5/I/3

(C) 2009 Davis et al; licensee BioMed Central Ltd.

This is an Open Access article distributed under the terms of the Creative Commons Attribution License (http://creativecommons.org/licenses/by/2.0), which permits unrestricted use, distribution, and reproduction in any medium, provided the original work is properly cited.

\begin{abstract}
Generating and identifying transformants is essential for many studies of gene function. In Arabidopsis thaliana, a revolutionary protocol termed floral dip is now the most widely used transformation method. Although robust, it involves a number of relatively time-consuming and laborious steps, including manipulating an Agrobacterium tumefaciens culture and aseptic procedures for the selection of plant lines harboring antibiotic-selection markers. Furthermore, where multiple transgenes are to be introduced, achieving this by sequential transformations over multiple generations adds significantly to the time required. To circumvent these bottlenecks, we have developed three streamlined sub-protocols. First, we find that $A$. thaliana can be transformed by dipping directly into an $A$. tumefaciens culture supplemented with surfactant, eliminating the need for media exchange to a buffered solution. Next, we illustrate that $A$. thaliana lines possessing a double-transformation event can be readily generated by simply by floral-dipping into a mixture of two A. tumefaciens cultures harboring distinct transformation vectors. Finally, we report an alternative method of transformant selection on chromatography sand that does not require surface sterilization of seeds. These sub-protocols, which can be used separately or in combination, save time and money, and reduce the possibility of contamination.
\end{abstract}

\section{Introduction}

The generation of transgenic plants has allowed for new insights into gene function. The creation of the floral-dip protocol [1] markedly advanced the ease of creating transformants in Arabidopsis thaliana. Transformation can now be performed at very large scales leading to near saturating mutagenesis $[2,3]$. It has also allowed the systematic study of gene function through transgenic approaches. Nevertheless, there is room to improve this protocol.

There are three steps that we find to be both time-consuming and costly. These are, firstly, the step following growth of the Agrobacterium tumefaciens (recently reclassified as Rhizobium radiobacter [4]) strain in liquid culture. Here, the protocol calls for pelleting the culture and resuspending in a buffered media. This typically takes one hour after the time spent preparing the buffered media (itself a time and cost consuming process). A second time-consuming step occurs when two separate transgenic constructs are to be introduced into a single A. thaliana line. This is accomplished by transformation with the first construct, followed by "stacking" the second transgene, either by transforming the first transgenic line with a second construct, or by crossing two independently derived trans- 
genic lines and obtaining the resultant F1 progeny. Both approaches are multi-generational to obtain the double transgenic plant, representing a significant time cost. Thirdly, in the selection of marker genes from transgenic seed in a population dominated by non-transformed lines, existing protocols for selection of lines typically requires surface sterilization of seed (a time-consuming process) and the plating of these seed on a sterile agar substrate (a costly process).

Clearly there is scope for improvements in steps within the floral-dip protocol. Evidence for this can be found in recently published protocols that target improvements in various stages of the transformation process [5-9]. Here, we present our simplified methods that are robustly effective in the generation and selection of transgenic $A$. thaliana plants. We provide a description of a bacterial-growth media that supports direct dipping and plant transformation after the trivial addition of surfactant, thereby eliminating the need to exchange bacteria from growth media to a buffer. Another improvement is a sub-protocol that allows for the simple introduction of two separate transgenes in one plant generation. Finally, we provide a subprotocol that eliminates the need to select on sterile conditions with the discovery that chromatography sand is a suitable alternative to an agar substrate during the seedselection process. Together, or separately, each of these sub-protocols offers time and cost savings to floral-dip transformation of $A$. thaliana.

\section{Materials and methods}

\section{Agrobacterium tumefaciens (recently reclassified as} Rhizobium radiobacter) cultures and culturing methods

A. tumefaciens strains ABI and GV3101 were both obtained from the Amasino group (University of Wisconsin-Madison, WI, USA). Bacterial transformation was as described [10]. The vectors respectively described were CCR2:LUC-HygR with the hpt-resistance gene conferring plant resistance to hygromycin [11], CAB2:LUC with the nptII-resistance gene conferring plant resistance to kanamycin [12], CCR2:LUC-Gent with the aacCl-resistance gene conferring plant resistance to gentamicin [13], and GI:LUC-Basta with the BAR-resistance gene conferring plant resistance to phosphinotricin [14]. To generate vectors for the identification of double transformation events, the FRB/Nluc and FKBP/CLuc elements were amplified by PCR from original plasmids [15] with KpnI and SacI restriction sites in the primers, and the resultant PCR products were digested with $\mathrm{KpnI}$ and SacI and the FRB/NLuc fragment was inserted into the KpnI and SacI sites of pPZP211 [16], and FRB/NLuc was similarly cut and ligated into the KpnI and SacI sites of pPZP221 [16]. For FRB/Nluc, the amplification primers were ATGGTACCATGGAGATGTGGCATGAAGG and ATTCAGAGCTCTCCATCCTTGTCAATCAAGGCG, and for FKBP/CLuc, the primers were ATGGTACCCTCGAGCCGCGGACTAGTATGTCCGGTTATGTA-AACAA and ATTCAGAGCTCTCATTCCAGTTTTAGAAGCTC.

Bacteria were generally grown in liquid culture at $28^{\circ} \mathrm{C}$, 250 rpm, in YEBS liquid media ( $1 \mathrm{~g} / \mathrm{L}$ yeast extract, $5 \mathrm{~g} /$ $\mathrm{L}$ beef extract, $5 \mathrm{~g} / \mathrm{L}$ sucrose, $5 \mathrm{~g} / \mathrm{L}$ bacto-peptone, $0.5 \mathrm{~g} / \mathrm{L}$ magnesium sulphate; adjusted $\mathrm{pH} 7$ ). LB media was also used (10 g/L bacto-tryptone, $5 \mathrm{~g} / \mathrm{L}$ yeast extract, $5 \mathrm{~g} / \mathrm{L}$ sodium chloride; adjusted pH 7.5). Appropriate antibiotics were added to the respective cultures.

\section{PCR conditions}

To detect the presence of FRB DNA, PCR was performed with the primers GAAGAGGCATCTCGTTTGTA and TAATAGAGGTCCCAGGCTTG. The product size was 221 bp. To detect the presence of FKBP DNA, PCR was performed with the primers GGGGCGGAGTGCAGGTGGAA and AAGACGAGAGTGGCATGTGG. The product size was 300 bp. The multiplex PCR involved the inclusion of the four above primers, with the appropriate plant DNA extracted as described [17]. PCR was as standard, and DNA presence was detected after electrophoresis on a $2 \%$ agarose gel.

\section{Arabidopsis thaliana growth}

Flowering plants were grown essentially as described [1]. Selection of transgenic seeds on agar was on solid MS3 media [4.4 g/L Murashige \& Skoog Basal Salt mixture (Sigma-Aldrich cat: M5524), $30 \mathrm{~g} / \mathrm{L}$ sucrose, $0.5 \mathrm{~g} / \mathrm{L}$ Monohydrate 2-(N-morpholino)ethanesufonic acid (MES); adjusted pH 5.7, 1.5\% phyto agar (Duchefa Biochemie)]. Seed sterilization was as described [1], except that a 33\% commercial bleach/Triton X-100 solution was used [33\% DanKlorix (this is $28 \mathrm{~g} / \mathrm{L}$ sodium hypochlorite; Colgate-Palmolive; Hamburg) and $200 \mu \mathrm{L} / \mathrm{L}$ Triton X100].

Selection on sand in a plastic-petri plate started with saturating chromatography sand with liquid MS0 media (1.1 $\mathrm{g} / \mathrm{L}$ MS basal salt, $0.5 \mathrm{~g} / \mathrm{L}$ MES; adjusted $\mathrm{pH} 5.7)$. The sands reported here were i) Silicon dioxide ( $\mathrm{SiO} 2)$; purum p.a.; acid purified; 40-200 mesh (84880 - Fluka), and ii) Quartz (SiO2); purum p.a.; powder; < 230 mesh (00653 - Sigma). Appropriate antibiotics were added to the MS0 solution before sand saturation. Dry seeds were sprinkled onto the wet sand, and after a two-day treatment at $4{ }^{\circ} \mathrm{C}$, plants were allowed to grow for two weeks.

\section{Protocols \\ Sub-protocol I: simplification of the bacterial dipping medium \\ Background}

The original "vacuum infiltration" protocol begins with a physical introduction of bacteria to the flower. For this, an 
A. tumefaciens culture with saturated cell density is exchanged to a buffered media, and this is applied to flowering plants under a weak vacuum [18]. Clough and Bent (1998) developed a refined systematic protocol that eliminated the need for vacuum introduction of bacteria. This led to a substitution of the buffered media. These authors found that inclusion of sucrose in the buffered media was critical, but many other ingredients present in the "vacuum infiltration" media formulation could be omitted. Importantly in this floral-dip protocol, the inclusion of the surfactant Silwet L77 was found to be required if a vacuum-pressure was not used [1].

We sought to further simplify the dipping recipe by testing if A. tumefaciens grown in a standard bacterial media (YEBS) (recipe listed below) would suffice for dipping after the supplement of sucrose and Silwet L77. We found that after saturated growth in YEBS media of A. tumefaciens cell line ABI harboring the CCR2:LUC transgene [11], the addition of both $25 \mathrm{~g}$ per L of sucrose and $200 \mu \mathrm{L}$ Silwet L77 per L of culture supported robust transfomation (Figure 1). Furthermore, the popular A. tumefaciens GV3101 cell line harboring the above-mentioned CCR2:LUC transgene was also robustly effective in transformation after growth in YEBS. As many labs use LB media, we tested the suitability of this media variation. Whereas the rate of transformation of $A$. thaliana transgenics was significantly lower from A. tumefaciens grown in LB media supplemented with $25 \%$ sucrose (w/v) and $0.02 \%$ Silwet L77, this preparation was also found to support the generation of transgenic plants (Figure 1). As another example, we transformed the above A. tumefaciens cell line GV3101 harboring the CCR2::LUC construct into a variety of $A$. thaliana accessions. Resultant transformation rates at the $\mathrm{T} 1$ generation were: Col-0, 1.3\%; Esp-1, 0.25\%; Jm-1, 0.7\%; En-1, 0.6\%; No-0, 0.3\%; Oy-0, 0.1\%; RLD, 0.9\%; Tanz- $1,0.5 \%$. Consistently and routinely, from an array of transformation constructs, and an array of A. thaliana genotypes and accessions, robust transformation rates between $0.1 \%$ and $10 \%$ have been observed. To date, thousands of construct/accession combinations have been dipped with the above recipe, in dozens of labs, and we have never encountered a failure in transformant identification as a result of the direct-dip protocol.

Motivated from the multiple media formulations that facilitated transformation, we tested whether the addition of Silwet L77 in the absence of supplemented sucrose would also be effective to generate transgenic plants after dipping, and found that simply adding the detergent to the bacteria culture, when grown in YEBS, was sufficient for floral-dip transformation (data not shown, and below protocol \#2, and Figure 2). Overall, the transformation rates reported here were similar to the transformation yield of the traditional floral-dip protocol $[1,6,9]$. As such,

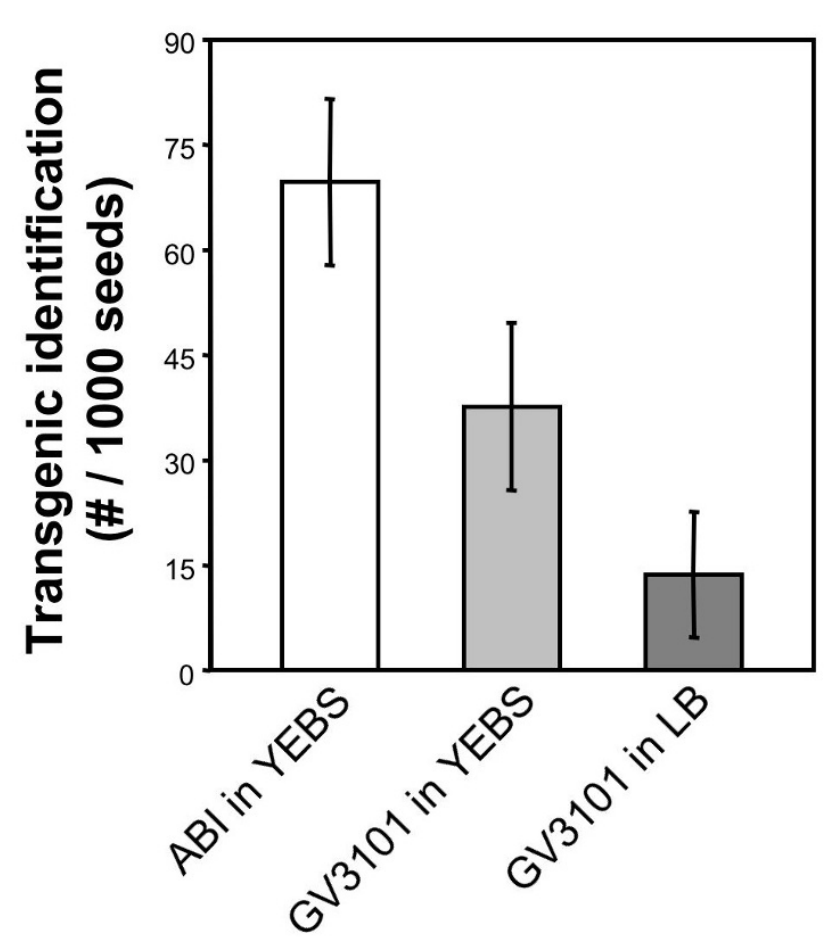

Figure I

Transformation frequencies detected after application of the 'direct dip' protocol. A. tumefaciens strains $A B I$ and GV3IOI harboring the CCR2:LUC-HygR transgene were cultured on YEBS or LB liquid media as indicated. Floral dipping of $A$. thaliana plants was subsequently performed according to the 'direct dip' protocol, involving supplementation of the media with surfactant and sucrose. Selection of TI seedlings was on solid MS3 medium containing hygromycin.

we now consider the need to exchange growth media to a buffered solution to be entirely eliminated.

Direct dip protocol

This protocol presupposes that a suitable transgenic $A$. tumefaciens cell line has already been generated. If assistance is needed on how to generate a construction for plant transformation, or how to transform A. tumefaciens with this vector, we direct the reader to $[8,10,19]$.

1. Identify transformed A. tumefaciens cells from an agar plate.

2. Start a $50 \mathrm{~mL}$ culture in YEBS with a bacterial streak from this transformation. Use appropriate antibiotics for bacterial selection of the Ti-plasmid and the introduced transformation vector. Grow this culture at $28-30^{\circ} \mathrm{C}$ until cell density is saturated (typically $2-3$ days). NOTE:Room temperature growth on a rotating lab shaker is possible if an incubator is not available. 


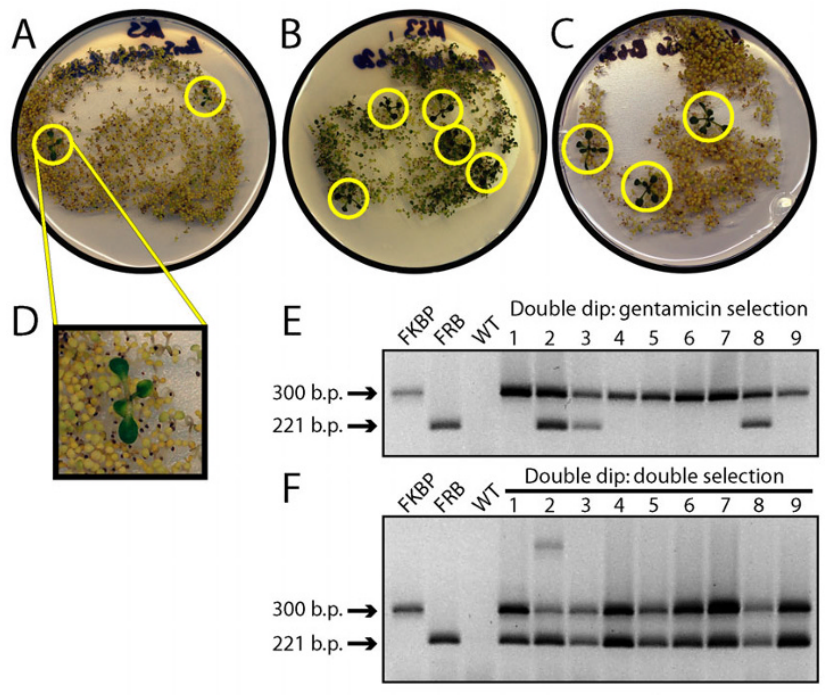

Figure 2

Identification of doubly transformed $A$. thaliana lines generated using the 'double dip' protocol. A: Growth of $A$. thaliana seedlings on MS3 plates containing both gentamicin $(100 \mu \mathrm{g} / \mathrm{mL})$ and kanamycin $(50 \mu \mathrm{g} / \mathrm{mL})$. Seeds were harvested from a mother plant that had simultaneously been transformed with respective $A$. tumefaciens $A B I$ lines separately harboring PPZP2 I I-FRB/NLuc (gentamicin-resistance) and PPZP22I-FRB/NLuc (kanamycin-resistance). B: Growth of a replicate batch of double-dipped $A$. thaliana seedlings on gentamicin alone. C: Growth of a replicate batch of doubledipped A. thaliana seedlings on kanamycin alone. Circles in A$C$ indicate antibiotic resistant plants. D: An expanded view of a robust seedling growing on both gentamicin and kanamycin. E: Multiplex genomic PCR of FRB and FKBP sequences in genomic DNA from nine lines selected on gentamicin alone. F: Multiplex genomic PCR of FRB and FKBP sequences in nine lines selected on both antibiotics. "FKB" indicates the PCR product obtained from a known kanamycin-resistant transgenic line; "FRB" indicates the PCR product obtained from a known gentamicin-resistant transgenic line; "WT" represents the negative control using a non-transgenic line.

3. Pour the entire $50 \mathrm{~mL}$ culture into $450 \mathrm{~mL}$ of YEBS. Further antibiotics for bacterial selection are not required, but addition will not interfere with subsequent steps. Grow for 8 hours NOTE:Start the $500 \mathrm{~mL}$ culture in the morning and it will be ready for plant dipping just before the end of the working day.

4. Add and mix 100-200 $\mu \mathrm{L}$ Silwett L77 to the culture; volume of surfactant is plant-genotype dependent. NOTE: if a larger volume of A. tumefaciens-dipping solution is needed, the final saturated culture can be diluted to $1 \mathrm{~L}$ with distilled water supplemented with 5\% $(w / v)$ sucrose concurrent with doubling the amount of Silwet L77. No effects on transformation efficiency have been noted.
5. This simple solution is ready for dipping of $A$. thaliana plants. Briefly, grow 6-10 plants per pot in $2.5 \mathrm{l} / 2.5$ " pots, or any other suited growing container. Dip A. thaliana lines that have bolted, where visible flowers are present. Recent papers graphically illustrate this dipping process $[7,9]$. NOTE:in slightly over-grown plants, existing siliques can be excised before dipping.

6. Seal plants in closed plastic bags overnight to increase humidity, which promotes transgenic yield. Remove plants from their sealed environment before 24 hours have passed. It is critical that the enclosure is not left over 24 hours, as extended humidity in the presence of $A$. tumefaciens leads to plant death. NOTE: as previously noted [1], plants can be re-dipped every 3-5 d resulting an increase in transformation percentage, but a modest decrease in total seed yield. Our standard practice is to dip twice, with the four-day interval separating the two interventions.

7. Let the plants grow until dry seeds are ready for harvesting and collect these seeds.

8. Aseptically select seeds for transformation, as reported $[1,6,7,9]$, or under non-sterile conditions, as described below.

\section{Sub-protocol 2: simultaneous transformation of A. thaliana with two transformation vectors \\ Background}

The insertion of more than one transgenic construct into the A. thaliana genome is necessary for several in vivo assays of protein interaction, such as BRET, FRET, and protein-fragment-complementation assays (PCA), and colocalization studies using fluorescent proteins [20-23]. Reconstruction of biochemical pathways can also require multiple tranformation events [24]. For these reasons, we sought to decrease the time to generate doubly transformed A. thaliana lines after the above "direct dip." We describe here a simple floral-dipping sub-protocol by which two separate transgenes can be inserted to a plant in a single $A$. thaliana generation, thus saving considerable time and resources.

Floral-dipping with a mixture of two separately grown $A$. tumefaciens cultures, each harboring a different transformation construct, can generate double transformants. Shown in Figure 2 are T1 transformants selected on media containing two selection agents, one for each construct. Note that in T1 seed from a double-dipped plant where only one transgene is selected for, double transgenics were still routinely detected (Figure 2E). When such T1 seed were subjected to double selection, only double transformants were found (Figure 2F). Not surprisingly, the rate of double transformation events is less frequent than single-transformation events, by a factor of $\sim 4-8$ (data not 
shown). We cannot speculate on why the double transformation rate is this high. The rate is more than sufficient to obtain an adequate number of double transformants for further analysis.

\section{Double dip Protocol}

1. Transform and individually select under antibiotic selection two A. tumefaciens lines each harboring the relevant vector. As for an equivalent "single dipping" method performed in series, each vector should confer a different resistance gene to the plant.

2. Prepare the two A. tumefaciens cultures by the method outlined above. If a final volume of $500 \mathrm{~mL}$ is required, add the starter $50 \mathrm{~mL}$ culture to $200 \mathrm{~mL}$ YEBS for $\sim 8 \mathrm{~h}$ growth, giving a total volume of $250 \mathrm{~mL}$ for each individual culture. The cultures are not mixed at this stage, and are grown in separate culture flasks.

3. Mix the A. tumefaciens cultures together, resulting in $500 \mathrm{~mL}$ of bacterial cells in YEBS. Add 100-200 $\mu \mathrm{L}$ Silwet77 , as described above.

4. Proceed with floral dipping according to the method previously outlined above.

5. Select for double transformants on a suitable support substrate containing both of the relevant antibiotics/herbicides at appropriate selective concentrations.

\section{Sub-protocol 3: selecting A. thaliana transgenics on sand Background}

One limitation in the identification of transgenic A. thaliana lines after floral dip is that the seeds are often internally contaminated with the A. tumefaciens line used. Furthermore, there is often fungal contamination within the seed. For these reasons, we wished to establish a protocol for antibiotic selection that could be used under non-aseptic conditions. As an added benefit, this would eliminate the time needed to surface-sterilize seed prior to agar culture. In pilot experiments, we tried using various concentrations of antibiotics with soil-grown plants carrying different antibiotic-resistance genes. We were never successful in identifying transformants on soil. We wondered if a simpler substrate would suffice, and therefore, we attempted to select on chromatography paper $A$. thaliana transformed with the above described CCR2:LUC reporter line (which generates linked hygromycin resistance). This was successful (data not shown), but daily monitoring of water levels was required given the limited surface area of paper as a growth substrate. There was thus no attempt to establish a generic protocol for selection on chromatography paper. Nevertheless, we were confident that it was possible to select for an antibiotic resistance marker under non-sterile conditions.
To establish a general substrate for non-aseptic selection of transgenics, we reasoned that sand might be an alternative over paper. In pilot experiments of horticultural sand, we found considerable variability to establish a working protocol. In particular, it was noticed that pre-washing the sand improved the selection ability. This led us to test prewashed ground quartz, a chromatography sand that is readily available from a number of commercial sources. We tested a variety of grades and granulation sizes and found that dry quartz sand (Sigma catalogue no. 00653) or dry silicon dioxide (Fluka catalogue no. 84880) worked well for a wide range of antibiotic/herbicide selective agents. Shown in Figure 3 is the ability of four different marker genes to be selected on chromatography sand. The vectors used for this transformation experiment are described in the Methods section. To date, every selective agent tested was effective in transgenic identification on chromatography sand.

The described techniques for sand selection can be more easily scaled up compared to conventional transformant selection, simply by using more sand for larger screens that require more total seed. In particular, we note that
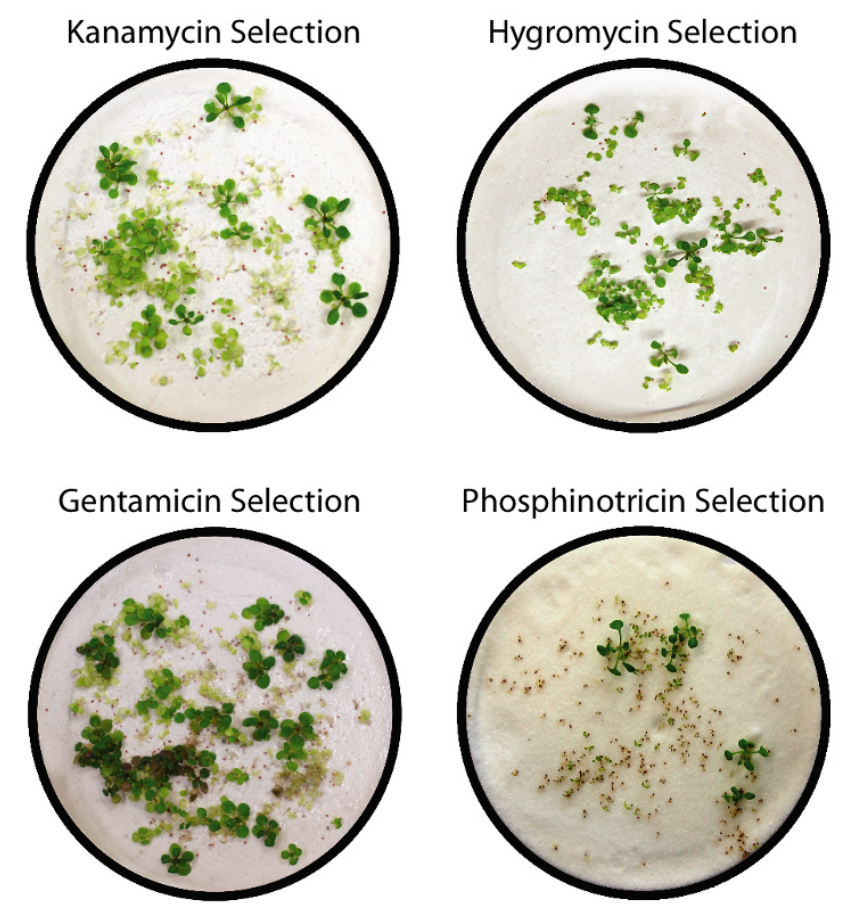

\section{Figure 3}

Selection of $A$. thaliana transformants on sand. The image shows seedlings grown on chromatography sand respectively under selection with kanamycin $(100 \mu \mathrm{g} / \mathrm{mL})$, hygromycin $(60 \mu \mathrm{g} / \mathrm{mL})$, gentamicin $(125 \mu \mathrm{g} / \mathrm{mL})$ and phosphinotricin $(12.5 \mu \mathrm{g} / \mathrm{mL})$. Note the readily identifiable transformants. 
chromatography sand can be purchased for a lower cost compared to agar substrates. Similarly, this selection technique can be used in classrooms that do not have access to sterile conditions. Finally, the success rate of transformant identification and resultant plant survival in the sand technique can be higher than that obtained from selection on sterile agar plates, because transformants are not readily contaminated with microorganisms.

\section{Sandy way Protocol}

1. Place $\sim 20 \mathrm{~mL}$ dry quartz sand (Sigma \#00653) or dry Silicon dioxide (Fluka \#84880) in a $94 \mathrm{~mm}$ Petri dish. NOTE:Alternative chromatography sands can work, but the specific type of sand matters a great deal, as some sands were found to be entirely unsuitable for certain antibiotic-selection regimes. The above two sands are our favorites, but by no means do these limit potential substrate choices. A given sand must be tested empirically within any given lab for suitability of use under the given selective agent. Generally, we found that the more 'white' the sand, the greater the number of selective agents capable of supporting transformant selection.

2. Saturate the sand by pipetting $10 \mathrm{~mL} 1 / 4 \mathrm{MS}$ Basal Salt media (without sucrose) that is buffered and $\mathrm{pH}$-adjusted containing the selecting antibiotic. NOTE: the range of antibiotic/herbicide added to the MS solution before saturating sand can often be up to twice that usually added for standard agar selection, but this must be empirically tested within the lab.

3. Evenly disperse the wet sand by gently tapping the Petri dish against the lab bench. This distributes the muddy sand mixture and releases trapped air bubbles. Then pipette or decant off excess liquid media such that the wet sand is no longer muddy.

4. Carefully tap up to $100 \mu \mathrm{L}$ dry seed onto the wet sand. NOTE:too much seed can result in unwanted fungal contamination, and furthermore, identification of transformants can be a problematic when within a dense seedling canopy.

5. Stratify plates at $4{ }^{\circ} \mathrm{C}$ for approximately two days, depending on genotype.

6. Move plates to a growth cabinet, as typical for agar selection. NOTE: surgical tape can be wrapped around the Petri dish to slow evaporation; do not use parafilm as the lack of sucrose in the sand requires that the plants are dependent on $\mathrm{CO}_{2}$ as a carbon source for growth.

7. Approximately every $3 \mathrm{~d}$, open the lid of the Petri dish and add 1 to $5 \mathrm{~mL}$ 1/4 MS Basal Salt media, or water, such that the plate is adequately wet. Do not over-water. NOTE:under some selection conditions it might be necessary to add a second round of antibiotic treatment. This must be established empirically.

8. After 10-14 d, transformed plants should be easily identifiable. NOTE:failure to successfully identify transgenics means that the concentration of the selective agent needs to be modified and/or a different sand substrate needs to be tested.

9. To remove selected transgenics, with their now sandembedded roots, gently pipette $\sim 10 \mathrm{~mL}$ water to the sand to mildly flood the plate. The selected transgenic seedlings can now be easily removed with forceps and transferred to soil for further growth. NOTE:we additionally use the sandselection method for generations after the T1 selection. As one example, we select transgenic F1 seeds on sand.

\section{Competing interests}

The authors declare that they have no competing interests.

\section{Authors' contributions}

SJD conceived the experiments. AMD developed the "sandy way" sub-protocol. AH and AJM developed the "direct dip" sub-protocol. CD developed the "double dip" sub-protocol. SJD wrote the paper.

\section{Acknowledgements}

We thank Nancy A. Eckardt and Brian G. Forde for editorial improvement of this paper. The work in the Davis group was supported by the Max Planck Society, the German Israeli Program (DIP: Cooperation; DIP project H 3.I), and the German Science Foundation (DFG: DAI06I/2-I). The Biotechnology and Biological Sciences Research Council funded work in the Millar group (BBSRC award E015263) and the Hall group (BBSRC award (F0053I8).

\section{References}

I. Clough SJ, Bent AF: Floral dip: a simplified method for Agrobacterium-mediated transformation of Arabidopsis thaliana. Plant J 1998, 16:735-743.

2. Rosso MG, Li Y, Strizhov N, Reiss B, Dekker K, Weisshaar B: An Arabidopsis thaliana T-DNA mutagenized population (GABI-Kat) for flanking sequence tag-based reverse genetics. Plant Mol Biol 2003, 53:247-259.

3. Alonso JM, Stepanova AN, Leisse TJ, Kim CJ, Chen H, Shinn P, Stevenson DK, Zimmerman J, Barajas P, Cheuk R, et al:: Genome-wide insertional mutagenesis of Arabidopsis thaliana. Science 2003, 30I:653-657.

4. Young JM, Kuykendall LD, Martinez-Romero E, Kerr A, Sawada H: A revision of Rhizobium Frank 1889 , with an emended description of the genus, and the inclusion of all species of Agrobacterium Conn 1942 and Allorhizobium undicola de Lajudie et al. 1998 as new combinations: Rhizobium radiobacter, $\mathbf{R}$. rhizogenes, R. rubi, R. undicola and R. vitis. Int J Syst Evol Microbiol 200I, 5 I (Pt I):89-103.

5. Chung MH, Chen MK, Pan SM: Floral spray transformation can efficiently generate Arabidopsis transgenic plants. Transgenic Res 2000, 9:47I-476.

6. Harrison SJ, Mott EK, Parsley K, Aspinall S, Gray JC, Cottage A: A rapid and robust method of identifying transformed Arabidopsis thaliana seedlings following floral dip transformation. Plant Methods 2006, 2:19.

7. Liu NY, Zhang ZF, Yang WC: Isolation of embryo-specific mutants in Arabidopsis: plant transformation. Methods Mol Biol 2008, 427:9I-100. 
8. Logemann E, Birkenbihl RP, Ulker B, Somssich IE: An improved method for preparing Agrobacterium cells that simplifies the Arabidopsis transformation protocol. Plant Methods 2006, 2:16.

9. Zhang X, Henriques R, Lin SS, Niu QW, Chua NH: Agrobacteriummediated transformation of Arabidopsis thaliana using the floral dip method. Nat Protoc 2006, I:64|-646.

10. Mattanovich D, Ruker F, Machado AC, Laimer M, Regner F, Steinkellner H, Himmler G, Katinger H: Efficient transformation of Agrobacterium spp. by electroporation. Nucleic Acids Res 1989, I 7:6747.

11. Doyle MR, Davis SJ, Bastow RM, McWatters HG, Kozma-Bognar L, Nagy F, Millar AJ, Amasino RM: The ELF4 gene controls circadian rhythms and flowering time in Arabidopsis thaliana. Nature 2002, 4l 9:74-77.

12. Millar AJ, Short SR, Chua NH, Kay SA: A novel circadian phenotype based on firefly luciferase expression in transgenic plants. Plant Cell 1992, 4: 1075-1087.

13. Farre EM, Harmer SL, Harmon FG, Yanovsky MJ, Kay SA: Overlapping and distinct roles of PRR7 and PRR9 in the Arabidopsis circadian clock. Curr Biol 2005, 15:47-54.

14. Ding Z, Millar AJ, Davis AM, Davis SJ: TIME FOR COFFEE encodes a nuclear regulator in the Arabidopsis thaliana circadian clock. Plant Cell 2007, 19:1522-1536.

15. Luker KE, Smith MC, Luker GD, Gammon ST, Piwnica-Worms H, Piwnica-Worms D: Kinetics of regulated protein-protein interactions revealed with firefly luciferase complementation imaging in cells and living animals. Proc Natl Acad Sci USA 2004, I0 I: I2288-12293.

16. Hajdukiewicz P, Svab Z, Maliga P: The small, versatile pPZP family of Agrobacterium binary vectors for plant transformation. Plant Mol Biol 1994, 25:989-994.

17. Michaels SD, Amasino RM: High throughput isolation of DNA and RNA in 96-well format using a paint shaker. Plant Molecular Biology Reporter 200I, 19:227-233.

18. Bechtold N, Ellis J, Pelletier G: In planta Agrobacterium-mediated gene transfer by infiltration of adult Arabidopsis thaliana plants. CR Acad Sci Paris Life Sci 1993, 3 I 6: I I 94-I I 99.

19. de la Riva GA, González-Cabrera J, Vázquez-Padrón R, Ayra-Pardo C: Agrobacterium tumefaciens: a natural tool for plant transformation. Electronic Journal of Biotechnology I998, I:I.

20. Bracha-Drori K, Shichrur K, Katz A, Oliva M, Angelovici R, Yalovsky $S$, Ohad N: Detection of protein-protein interactions in plants using bimolecular fluorescence complementation. Plant J 2004, 40:419-427.

21. Walter M, Chaban C, Schutze K, Batistic O, Weckermann K, Nake C, Blazevic D, Grefen C, Schumacher K, Oecking C, et al.: Visualization of protein interactions in living plant cells using bimolecular fluorescence complementation. Plant J 2004, 40:428-438.

22. $\mathrm{Xu} \mathrm{J}$, Scheres B: Dissection of Arabidopsis ADP-RIBOSYLATION FACTOR I function in epidermal cell polarity. Plant Cell 2005, 17:525-536.

23. Xu X, Soutto M, Xie Q, Servick S, Subramanian C, von Arnim AG, Johnson $\mathrm{CH}$ : Imaging protein interactions with bioluminescence resonance energy transfer (BRET) in plant and mammalian cells and tissues. Proc Natl Acad Sci USA 2007, 104: 10264-10269.

24. Burgal J, Shockey J, Lu C, Dyer J, Larson T, Graham I, Browse J: Metabolic engineering of hydroxy fatty acid production in plants: RcDGAT2 drives dramatic increases in ricinoleate levels in seed oil. Plant Biotechnol J 2008, 6:819-83I.

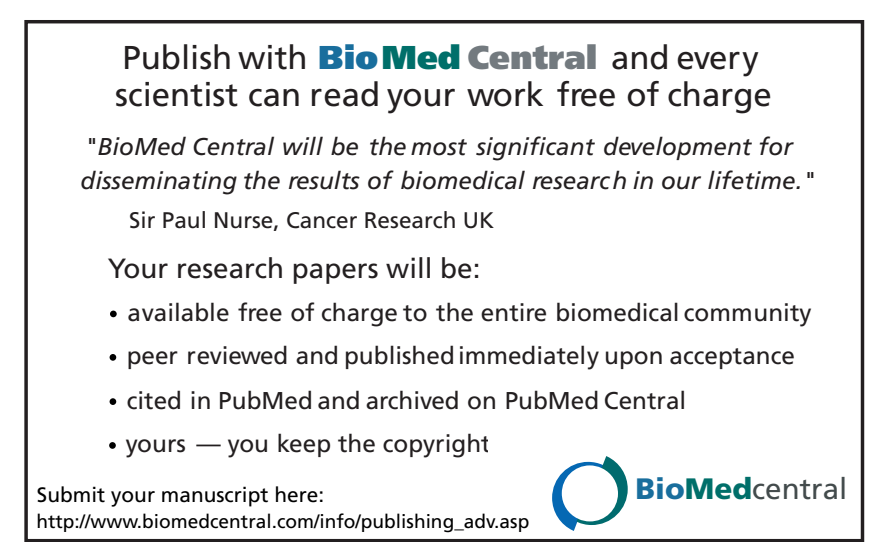

